\title{
Global Trends and Regional Differences in Hepatitis C Virus Infection Prevalence and Implications for Prevention — Worldwide, 1990-2017
}

\author{
Wenzhan Jing'; Jue Liu'; Min Liu ${ }^{1, *}$
}

\section{Summary}

What is already known on this topic?

The global prevalence of hepatitis $\mathrm{C}$ virus (HCV) infection in 2015 has been modeled to assess the disease burden in 21 Global Burden of Disease (GBD) regions. However, there is no study to clarify the global long-term trends and regional differences in $\mathrm{HCV}$ infection prevalence.

\section{What is added by this report?}

This report clarified the global temporal trends and regional differences in $\mathrm{HCV}$ infection prevalence. Global HCV infection age-standardized prevalence rate (ASR) gradually decreased from 1990 to 2017 except in Eastern Europe, and liver cancer due to hepatitis C ASR increased worldwide with drastic shifts in lowmiddle Socio-Demographic Index (SDI) regions in the last decade.

What are the implications for public health practice?

$\mathrm{HCV}$ is still prevalent worldwide despite the development of highly effective direct-acting antivirals (DAAs) and showed a reemergence concurrent with the opioid crisis. $\mathrm{HCV}$ infection prevention might involve at least 3 aspects: first, prohibiting HCV widespread transmission among general populations; second, increasing global DAAs coverage; and third, continuously investing in the development of the $\mathrm{HCV}$ vaccine.

Hepatitis $\mathrm{C}$ virus (HCV) infection is a major public health problem worldwide (1). It is necessary to clarify the global trends and regional differences in HCV infection prevalence to make tailored prevention strategies. The HCV infection burdens from 1990 to 2017 were collected from the Global Burden of Disease (GBD) Study 2017. Relative changes in HCV cases and estimated annual percentage changes (EAPCs) of age-standardized prevalence rate (ASR) with $95 \%$ confidence intervals $(\mathrm{CIs})$ were used to quantify temporal trends of $\mathrm{HCV}$ infection. $\mathrm{HCV}$ infection ASR decreased by an average of $0.67 \%$ (95\% CI: $0.64 \%-0.70 \%$ ) per year from $2,043.42$ per 100,000 in 1990 to $1,728.04$ per 100,000 in 2017 , while the number of HCV cases increased by $28.82 \%$ from 105.15 million in 1990 to 135.45 million in 2017. Against the global background of HCV infection prevalence decreasing, the ASR increased in Eastern Europe (EAPC=0.78, 95\% CI: 0.56-0.99). Liver cancer due to hepatitis $\mathrm{C}$ increased drastically in the low-middle Socio-Demographic Index (SDI) regions in the last decade. There is no vaccine for hepatitis $\mathrm{C}$, therefore, prevention should be focused on reducing exposure risk to $\mathrm{HCV}$ by safe injections, harm reduction, screening, and treatment.

About $55 \%-85 \%$ of infected persons develop chronic HCV infection without treatment, and $15 \%-30 \%$ of those with chronic infections develop complications within 20-30 years (2). Global incidence of $\mathrm{HCV}$ infection was 23.7 per 100,000 population in 2015 with 1.75 million new infections (3). About 71.1 million people were living with HCV, accounting for $1 \%$ of the population (1), and approximately 399,000 people died from cirrhosis and hepatocellular carcinoma (HCC) due to hepatitis $\mathrm{C}$ in 2016 (2). A watershed moment came in 2014 with the development of highly effective direct-acting antivirals (DAAs) for HCV infections (4). DAAs can not only cure those treated, thereby reducing death risk from cirrhosis and HCC, but also reduce HCV transmission and therefore its prevalence (5). Advances in HCV therapeutics prompted World Health Organization (WHO) member states to set targets for HCV elimination. However, only $20 \%$ of persons with hepatitis $\mathrm{C}$ know their diagnosis, and $15 \%$ of those with known hepatitis $\mathrm{C}$ have been treated (1). The global epidemic may continue to expand in magnitude in the absence of scaled-up interventions because new infections outnumbered the individuals dying from end-stage HCV infections and those who were cured.

The HCV infection burden, including acute hepatitis $\mathrm{C}$, cirrhosis and other chronic liver diseases 
due to hepatitis $\mathrm{C}$, and liver cancer due to hepatitis $\mathrm{C}$ from 1990 to 2017 by age group, region, and country, was collected from GBD 2017 (G). Specific methods of the estimation process for $\mathrm{HCV}$ infection prevalence were described elsewhere (G). First, anti-HCV seroprevalence data from population-based studies and surveys were reviewed to estimate the acute hepatitis C prevalence using DisMod-MR 2.1 model. Second, total cirrhosis and total liver cancer prevalence were modelled using hospital data and cause-specific mortality rate estimates. Third, the proportions of cirrhosis and liver cancer due to underlying etiologies such as hepatitis B, hepatitis C, and alcohol use were estimated by meta-regression. Finally, the prevalence of cirrhosis and liver cancer due to hepatitis $\mathrm{C}$ was estimated according to total cirrhosis and total liver cancer prevalence and the proportion attributable to hepatitis C.

The 195 countries and territories were divided into 5 SDI regions according to total fertility rate under 25 years old, years of education for those aged 15 and older, and lag distributed income per capita and were separated into $21 \mathrm{GBD}$ regions based on their epidemiological homogeneity and geographical contiguity. The ASR calculated by the direct method and absolute number of HCV cases were used to show $\mathrm{HCV}$ epidemic status. Relative changes in HCV cases and EAPCs of ASR with $95 \%$ CIs were calculated to quantify the temporal trends of $\mathrm{HCV}$ infection from 1990 to 2017. Change in HCV cases was defined as $\frac{\mathrm{HCV} \text { cases }_{2017}-\mathrm{HCV} \text { case }_{1990}}{\mathrm{HCV} \text { cases }_{1990}} \times 100 \%$. A regression line was fitted to the natural logarithm of ASR, for instance, $\quad y=\alpha+\beta x+\varepsilon$, where $y=\ln ($ ASR $)$ and $\mathrm{x}=$ calendar year, $\mathrm{EAPC}=100 \times\left(\mathrm{e}^{\beta}-1\right)$. All statistical analyses were conducted by $\mathrm{R}$ statistical software (version 3.5.1; The $\mathrm{R}$ Foundation for Statistical Computing, Vienna, Austria). Statistical significance was attributed to $p$ values $(p<0.05)$.

The HCV infection ASR was unevenly distributed worldwide with the highest in Egypt (Figure 1). HCV cases in China and India accounted for one third of the global $\mathrm{HCV}$ infections. The $\mathrm{HCV}$ infection ASR decreased by $0.67 \%$ (95\% CI: $0.64 \%-0.70 \%$ ) per year globally from 2043.42 per 100,000 in 1990 to 1728.04 per 100,000 in 2017 (Table 1); while the number of HCV cases increased by $28.82 \%$ from 105.15 million in 1990 to 135.45 million in 2017, and increased significantly among individuals aged 50-69 years old and 70 years old above by $63.87 \%$ and
76.53\%, respectively (Supplementary Figure S1 available in http://weekly.chinacdc.cn/). Against the global trend of ASR falling, an increasing trend was reported in Eastern Europe (EAPC $=0.78,95 \%$ CI: 0.56-0.99). Meanwhile, the number of HCV cases only decreased in Central and Western Europe. Over 99\% of HCV infections were cirrhosis and other chronic liver diseases (Supplementary Figure S1), and the global trends and regional differences of which were similar to those of global HCV infections (Supplementary Figure S2 available in http://week ly.chinacdc.cn/).

The highest acute hepatitis C ASR was observed in Central Asia and the lowest in Western Europe (Supplementary Figure S3 available in http://weekly. chinacdc.cn/). The acute hepatitis C ASR decreased by $0.62 \%$ (95\% CI: $0.59 \%-0.65 \%$ ) per year from 9.50 per 100,000 in 1990 to 8.05 per 100,000 in 2017; while the number of acute hepatitis $\mathrm{C}$ cases increased by $8.63 \%$ from 0.54 million in 1990 to 0.59 million in 2017. The acute hepatitis C ASR increased in Eastern Europe (EAPC $=0.65$ 95\% CI: $0.45-0.86$ ), and the number of acute hepatitis $\mathrm{C}$ cases decreased distinctly in Central Europe (30.47\%) and East Asia (22.5\%) (Supplementary Figure S3).

Liver cancer due to hepatitis C ASR was distributed heterogeneously worldwide with the highest in highincome Asia Pacific Region (14.39 per 100,000 in 2017) (Figure 2). Globally, liver cancer due to hepatitis C ASR increased by $0.87 \%$ (95\% CI: $0.80 \%-0.94 \%$ ) per year from 3.38 per 100,000 in 1990 to 4.54 per 100,000 in 2017. Meanwhile, the number of liver cancer due to hepatitis C increased by $159.40 \%$ from 0.14 million in 1990 to 0.37 million in 2017, and significantly increased by $253.15 \%$ among those over 70 years old (Supplementary Figure S1). Remarkably, liver cancer due to hepatitis C ASR increased drastically in low-middle SDI regions in the last decade, and increases were also observed in middle SDI regions (Supplementary Figure S4 available in http://weekly.chinacdc.cn/). The liver cancer due to hepatitis C ASR increased enormously in Australasia (EAPC $=4.66,95 \%$ CI: 4.24-5.08) and high-income North America (EAPC $=4.47$, 95\% CI: 3.99-4.95), and the number of cases also increased by $521.99 \%$ and $455.62 \%$, respectively (Figure 2).

\section{DISCUSSION}

HCV infection ASR decreased globally except in 


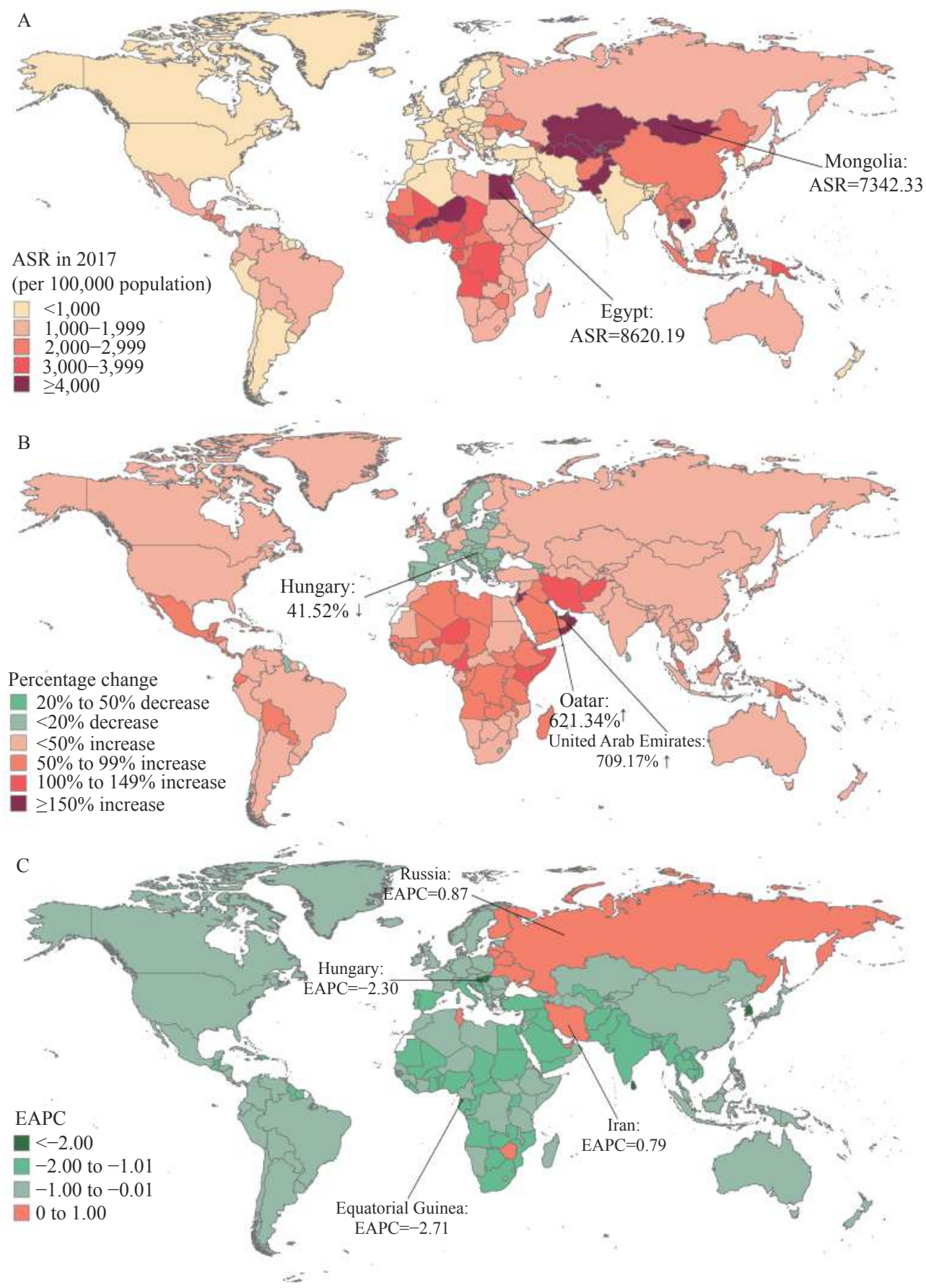

FIGURE 1. The global trends of HCV infection burden in 195 countries and territories. (A) The age-standardized prevalence rate (ASR) of HCV infection in 2017; (B) The percentage change in HCV cases between 1990 and 2017; (C) The estimated annual percentage changes (EAPCs) of age-standardized prevalence rate of HCV infection from 1990 to 2017.

Eastern Europe, and liver cancer due to hepatitis C ASR increased worldwide with drastic shifts in lowmiddle SDI regions in the last decade. The reduction of HCV infection ASR was partly due to the increased mortality due to liver-related causes and an ageing population. Similar with previous studies, Egypt had the highest HCV prevalence with $6.3 \%$ of the population living with $\mathrm{HCV}$ due to endemic schistosomiasis treatment by unsafe healthcare injections from the 1950s to 1980 s, and subsequently inadequate infection control and interfamilial transmission (7). Over 1 million people in Egypt, the first country to negotiate an alternative price of DAAs, were treated from 2015 to 2017; however, treatment 
TABLE 1. The estimated annual percentage changes (EAPCs) of the age standardized prevalence rate (ASR) of acute hepatitis C, cirrhosis, and other chronic liver diseases due to hepatitis C, liver cancer due to hepatitis C, and total HCV infections from 1990 to 2017 by region.

\begin{tabular}{|c|c|c|c|c|}
\hline \multirow[b]{2}{*}{ Characteristics } & \multicolumn{4}{|c|}{ EAPCs of ASR $(95 \% \mathrm{Cl})$} \\
\hline & Acute hepatitis C & $\begin{array}{l}\text { Cirrhosis and other } \\
\text { chronic liver diseases } \\
\text { due to hepatitis C }\end{array}$ & $\begin{array}{c}\text { Liver cancer due to } \\
\text { hepatitis } C\end{array}$ & Total HCV infections \\
\hline Global & $-0.62(-0.65$ to -0.59$)$ & $-0.67(-0.70$ to -0.64$)$ & $0.87(0.80$ to 0.94$)$ & $-0.67(-0.70$ to -0.64$)$ \\
\hline \multicolumn{5}{|l|}{ Socio-Demographic Index } \\
\hline Low & $-0.57(-0.64$ to -0.50$)$ & $-0.79(-0.83$ to -0.76$)$ & $-0.63(-0.67$ to -0.59$)$ & $-0.79(-0.82$ to -0.76$)$ \\
\hline Low-middle & $-0.59(-0.61$ to -0.56$)$ & $-0.87(-0.93$ to -0.82$)$ & $0.64(0.57$ to 0.71$)$ & $-0.87(-0.92$ to -0.82$)$ \\
\hline Middle & $-0.89(-0.93$ to -0.86$)$ & $-0.94(-0.98$ to -0.90$)$ & $0.19(-0.01$ to 0.38$)$ & $-0.94(-0.98$ to -0.89$)$ \\
\hline High-middle & $-0.78(-0.82$ to -0.73$)$ & $-0.61(-0.67$ to -0.56$)$ & $1.24(0.94$ to 1.53$)$ & $-0.61(-0.67$ to -0.55$)$ \\
\hline High & $-1.04(-1.12$ to -0.97$)$ & $-0.71(-0.76$ to -0.67$)$ & $1.34(1.13$ to 1.55$)$ & $-0.71(-0.75$ to -0.66$)$ \\
\hline \multicolumn{5}{|l|}{ Global Burden of Disease Region } \\
\hline High-income Asia Pacific & $-1.51(-1.64$ to -1.38$)$ & $-1.01(-1.06$ to -0.97$)$ & $-0.57(-0.75$ to -0.38$)$ & $-1.01(-1.05$ to -0.96$)$ \\
\hline Central Asia & $-0.34(-0.46$ to -0.23$)$ & $-0.63(-0.74$ to -0.52$)$ & $0.17(0.08$ to 0.26$)$ & $-0.63(-0.74$ to -0.52$)$ \\
\hline East Asia & $-0.66(-0.75$ to -0.57$)$ & $-0.67(-0.74$ to -0.6$)$ & $0.85(0.56$ to 1.13$)$ & $-0.67(-0.74$ to -0.59$)$ \\
\hline South Asia & $-0.68(-0.75$ to -0.61$)$ & $-1.04(-1.14$ to -0.94$)$ & $1.16(1.12$ to 1.20$)$ & $-1.04(-1.14$ to -0.94$)$ \\
\hline Southeast Asia & $-0.77(-0.79$ to -0.75$)$ & $-0.84(-0.86$ to -0.82$)$ & $0.09(0.00$ to 0.18$)$ & $-0.84(-0.86$ to -0.82$)$ \\
\hline Australasia & $-0.30(-0.36$ to -0.24$)$ & $-0.51(-0.57$ to -0.44$)$ & $4.66(4.24$ to 5.08$)$ & $-0.50(-0.56$ to -0.44$)$ \\
\hline Caribbean & $-1.07(-1.10$ to -1.03$)$ & $-1.00(-1.05$ to -0.95$)$ & $-0.51(-0.63$ to -0.39$)$ & $-1.00(-1.05$ to -0.95$)$ \\
\hline Central Europe & $-0.74(-0.87$ to -0.61$)$ & $-0.96(-1.02$ to -0.90$)$ & $0.57(0.40$ to 0.74$)$ & $-0.96(-1.02$ to -0.90$)$ \\
\hline Eastern Europe & $0.65(0.45$ to 0.86$)$ & $0.78(0.56$ to 1.00$)$ & $-0.18(-0.32$ to -0.04$)$ & $0.78(0.56$ to 0.99$)$ \\
\hline Western Europe & $-1.33(-1.44$ to -1.23$)$ & $-1.06(-1.13$ to -0.98$)$ & $2.38(2.02$ to 2.73$)$ & $-1.04(-1.12$ to -0.97$)$ \\
\hline Andean Latin America & $-0.51(-0.54$ to -0.47$)$ & $-0.69(-0.73$ to -0.65$)$ & $0.27(0.11$ to 0.44$)$ & $-0.69(-0.72$ to -0.65$)$ \\
\hline Central Latin America & $-0.94(-1.01$ to -0.86$)$ & $-0.63(-0.66$ to -0.60$)$ & $-0.63(-0.70$ to -0.57$)$ & $-0.63(-0.66$ to -0.60$)$ \\
\hline Southern Latin America & $-0.86(-0.93$ to -0.78$)$ & $-0.65(-0.71$ to -0.59$)$ & $-0.40(-0.52$ to -0.29$)$ & $-0.65(-0.71$ to -0.59$)$ \\
\hline Tropical Latin America & $-0.48(-0.64$ to -0.32$)$ & $-0.48(-0.69$ to -0.27$)$ & $0.00(-0.17$ to 0.17$)$ & $-0.48(-0.69$ to -0.27$)$ \\
\hline North Africa and Middle East & $-1.08(-1.12$ to -1.03$)$ & $-1.34(-1.43$ to -1.26$)$ & $1.10(0.91$ to 1.29$)$ & $-1.34(-1.42$ to -1.25$)$ \\
\hline High-income North America & $0.01(-0.08$ to 0.11$)$ & $-0.19(-0.34$ to -0.05$)$ & $4.47(3.99$ to 4.95$)$ & $-0.18(-0.32$ to -0.03$)$ \\
\hline Oceania & $-0.30(-0.39$ to -0.21$)$ & $-0.32(-0.38$ to -0.25$)$ & $0.32(0.27$ to 0.38$)$ & $-0.32(-0.38$ to -0.25$)$ \\
\hline Central Sub-Saharan Africa & $-0.64(-0.89$ to -0.40$)$ & $-0.70(-0.88$ to -0.52$)$ & $-1.22(-1.40$ to -1.04$)$ & $-0.70(-0.88$ to -0.52$)$ \\
\hline Eastern Sub-Saharan Africa & $-0.85(-0.95$ to -0.74$)$ & $-0.77(-0.87$ to -0.68$)$ & $-0.83(-0.95$ to -0.72$)$ & $-0.77(-0.87$ to -0.68$)$ \\
\hline Southern Sub-Saharan Africa & $-0.90(-1.08$ to -0.72$)$ & $-0.96(-1.12$ to -0.79$)$ & $-0.94(-1.41$ to -0.47$)$ & $-0.96(-1.12$ to -0.79$)$ \\
\hline Western Sub-Saharan Africa & $-0.99(-1.07$ to -0.92$)$ & $-1.07(-1.11$ to -1.02$)$ & $-1.17(-1.2$ to -1.14$)$ & $-1.07(-1.11$ to -1.02$)$ \\
\hline
\end{tabular}

numbers stagnated and finding those undiagnosed remained the biggest challenge (7). Being unaware of $\mathrm{HCV}$ infection can lead to serious health outcomes and increase HCV transmission risk (8). Consistent with this study, the WHO reported that hepatitis C was found in less than $0.5 \%$ of the population in Western, Northern, and Central Europe and as high as $3 \%-6 \%$ in many countries of Eastern Europe and Central Asia, and Eastern Europe suffered from an increasing trend of $\mathrm{HCV}$ infection due to sharing of needles and syringes (9).
Nowadays, even in areas where $\mathrm{HCV}$ infections were low, an increase in HCV transmission may occur (3). Consistent with our study, surveillance in the United States showed a reemergence of new HCV infections with reported acute hepatitis $\mathrm{C}$ rates increasing threefold from 0.3 per 100,000 population in 2009 to 1.2 per 100,000 population in 2018 concurrent with the nation's opioid crisis; and increasing rates among reproductive-aged persons put multiple generations at risk for chronic hepatitis $\mathrm{C}(8)$. Prevention should be focused on reducing exposure risk because no vaccine 

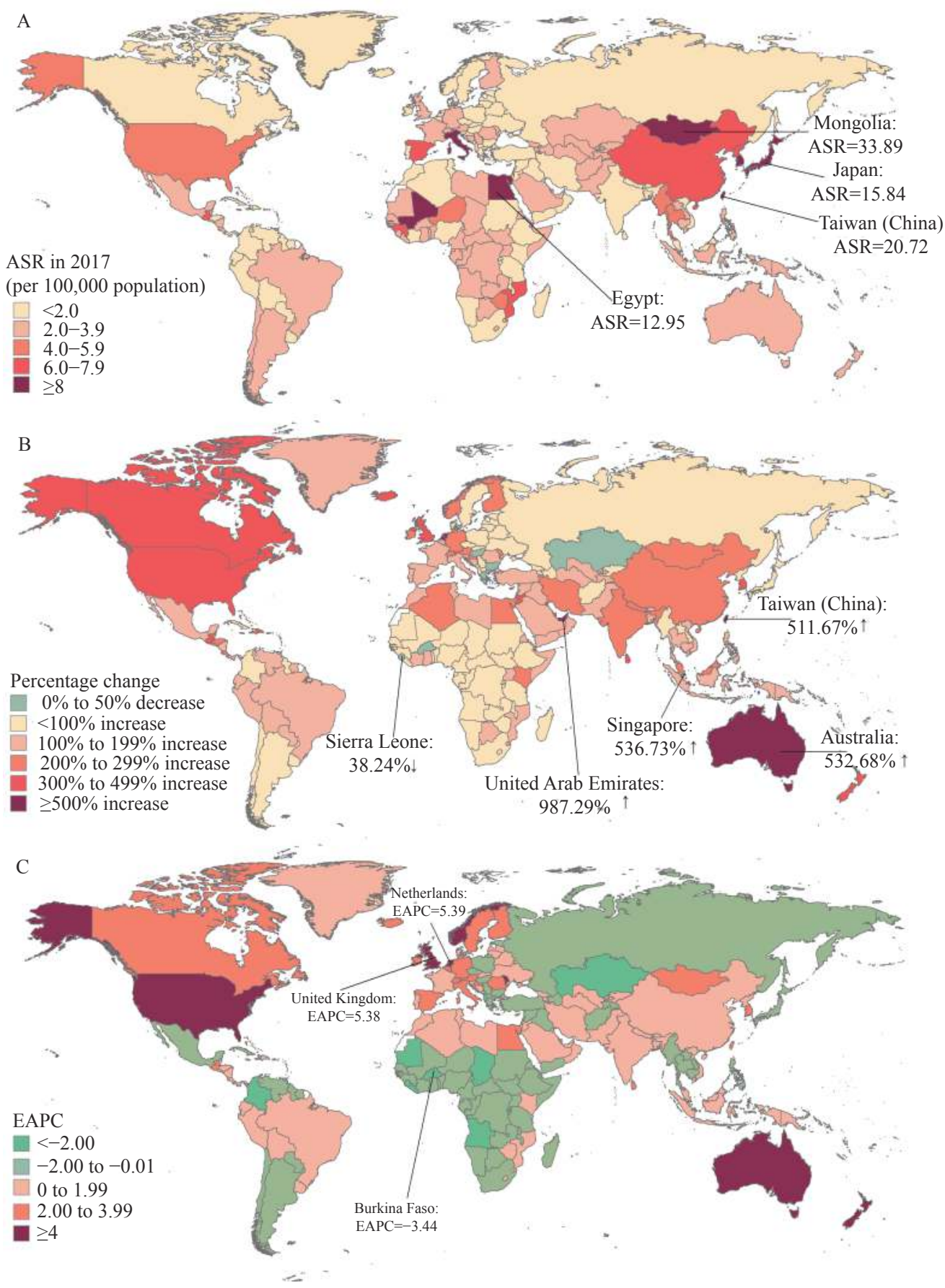

FIGURE 2. The global trends of liver cancer due to hepatitis $C$ in 195 countries and territories. (A) The age-standardized prevalence rate (ASR) of liver cancer due to hepatitis C in 2017; (B) The percentage change in the number of liver cancer due to hepatitis C between 1990 and 2017; (C) The estimated annual percentage changes (EAPCs) of age-standardized prevalence rate of liver cancer due to hepatitis C from 1990 to 2017.

exists for hepatitis C (9). The protection of safe injections and harm reduction and screening are key points to prevent new infections worldwide.

The global liver cancer due to hepatitis C ASR displayed increases and remarkably increased in most high-income regions probably due to the longer life expectancy and higher ageing population. To some extent, the increases were also driven by the increasing of obesity, diabetes, and steatosis, since these diseases could increase risk of advanced fibrosis among chronic HCV infections (10). A prospective cohort study reported DAA treatment reduced risk for mortality and HCC, therefore, DAAs should be considered in all chronic HCV infections (5). A global mathematical 
model estimated a comprehensive package of $\mathrm{HCV}$ prevention, screening, and treatment could result in a $61 \%$ reduction in mortality compared with the 2015 baseline (4). However, the prices of DAAs remained high in high-income countries and those middleincome countries where generic drugs were unable to access and licensing agreements were not covered (3).

To our knowledge, this is the first assessment of the global landscape, long-term trends, and regional differences in $\mathrm{HCV}$ infection prevalence using GBD study data. $\mathrm{HCV}$ is still prevalent worldwide despite the development of DAAs, and a reemergence of $\mathrm{HCV}$ was concurrent with the opioid crisis. HCV infection prevention might involve at least 3 aspects: first, prohibiting $\mathrm{HCV}$ widespread transmission among general populations; second, increasing global DAAs coverage; and third, continuously investing in the development of an HCV vaccine. Public health gains can be made in low-resource contexts, provided there is strong government will, budgetary commitments, and smart drug procurement to make life-saving treatments affordable.

Several limitations remained. First, availability of data and quality of available data in different regions might be incomparable and limit accuracy and robustness of $\mathrm{HCV}$ infection estimates in the modeling, evaluation of which was not listed due to limited space. Second, different regional governments had different priorities in HCV treatment, prevention, and healthcare policies. Third, EAPCs and change of HCV cases from 1990 to 2017 might mask the shortterm trends that reflected the effectiveness of recent prevention interventions.

Acknowledgements: We appreciate the works by the Global Burden of Disease study 2017 collaborators.

Fundings: This study was supported by the grant from National Natural Science Foundation of China
(71874003, 71934002, and 81703240).

doi: $10.46234 / \mathrm{ccdcw} 2020.151$

\# Corresponding author: Min Liu, liumin@bjmu.edu.cn.

${ }^{1}$ Department of Epidemiology and Biostatistics, School of Public Health, Peking University, Beijing, China.

Submitted: June 29, 2020; Accepted: July 16, 2020

\section{REFERENCES}

1. Spearman CW, Dusheiko GM, Hellard M, Sonderup M. Hepatitis C. Lancet 2019;394(10207):1451 - 66. http://dx.doi.org/10.1016/s01406736(19)32320-7.

2. World Health Organization. Fact-sheet: Hepatitis C. https://www.who. int/news-room/fact-sheets/detail/hepatitis-c. [2020-06-18].

3. World Health Organization. Global hepatitis report, 2017. http://www. who.int/hepatitis/publications/global-hepatitis-report2017/en/. [201803-20].

4. Heffernan A, Cooke GS, Nayagam S, Thursz M, Hallett TB. Scaling up prevention and treatment towards the elimination of hepatitis C: a global mathematical model. Lancet 2019;393(10178):1319-29. http://dx.doi.org/10.1016/s0140-6736(18)32277-3.

5. Carrat F, Fontaine H, Dorival C, Simony M, Diallo A, Hezode C, et al. Clinical outcomes in patients with chronic hepatitis $\mathrm{C}$ after directacting antiviral treatment: a prospective cohort study. Lancet 2019;393(10179):1453 - 64http://dx.doi.org/10.1016/s0140-6736(18) 32111-1.

6. GBD 2017 Disease and Injury Incidence and Prevalence Collaborators. Global, regional, and national incidence, prevalence, and years lived with disability for 354 diseases and injuries for 195 countries and territories, 1990-2017: a systematic analysis for the Global Burden of Disease Study 2017. Lancet 2018;392(10159):1789 - 858 http://dx.doi.org/10.1016/S0140-6736(18)32279-7.

7. World Health Organization. Egypt: 35 million people receive hepatitis C test. https://www.who.int/hepatitis/news-events/egypt-hepatitis-ctesting/en/. [2020-06-20].

8. Ryerson AB, Schillie S, Barker LK, Kupronis BA, Wester C. Vital signs: newly reported acute and chronic hepatitis $C$ cases - United States, 2009-2018. MMWR Morb Mortal Wkly Rep 2020;69(14):399 - 404. http://dx.doi.org/10.15585/mmwr.mm6914a2.

9. WHO Regional Office for Europe. Hepatitis $\mathrm{C}$ in the WHO European region. https://www.euro.who.int/_data/assets/pdf_file/0009/377253/ Fact-Sheet-Hepatitis-C_2019_ENG.PDF. [2020-06-20].

10. Dyal HK, Aguilar M, Bhuket T, Liu B, Holt EW, Torres S, et al. Concurrent obesity, diabetes, and steatosis increase risk of advanced fibrosis among HCV patients: a systematic review. Dig Dis Sci 2015;60(9):2813 - 24. http://dx.doi.org/10.1007/s10620-015-3760-3. 

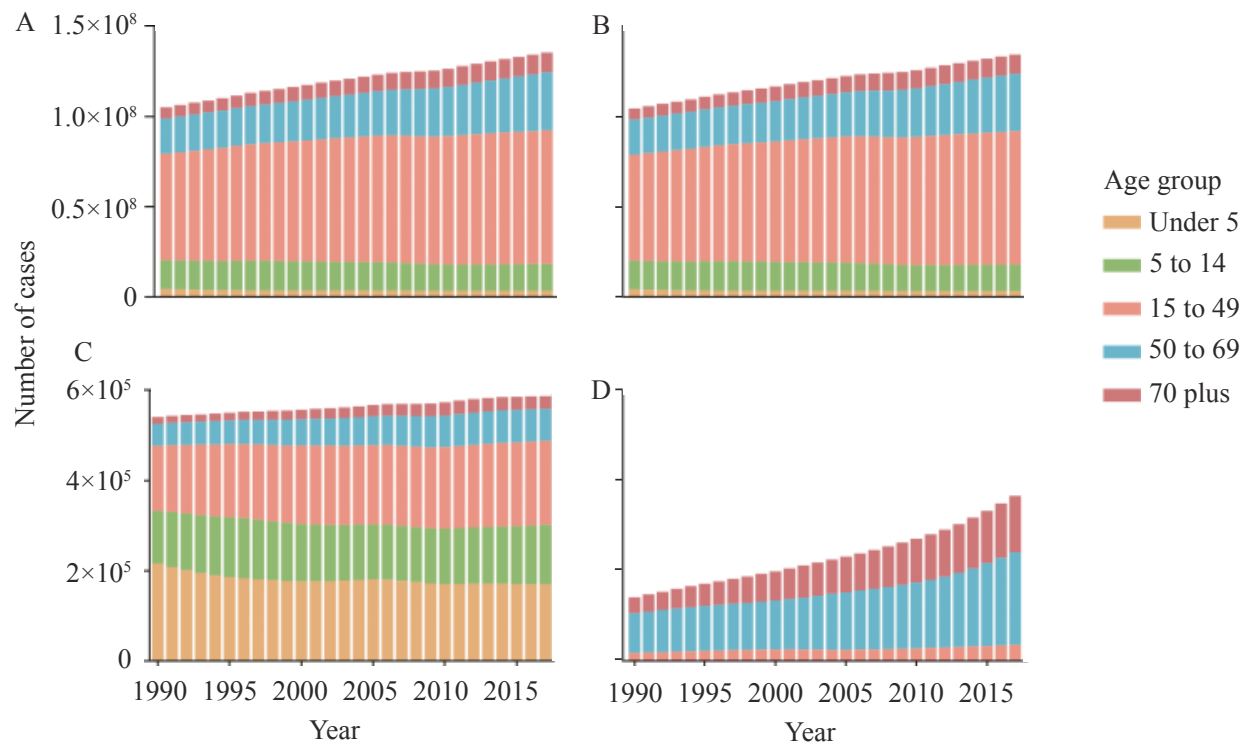

SUPPLEMENTARY FIGURE S1. The global burden of HCV infection by age group from 1990 to 2017, worldwide. (A) Total HCV cases. (B) Cirrhosis and other chronic liver diseases due to hepatitis C. (C) Acute hepatitis C. (D) Liver cancer due to hepatitis $\mathrm{C}$. 

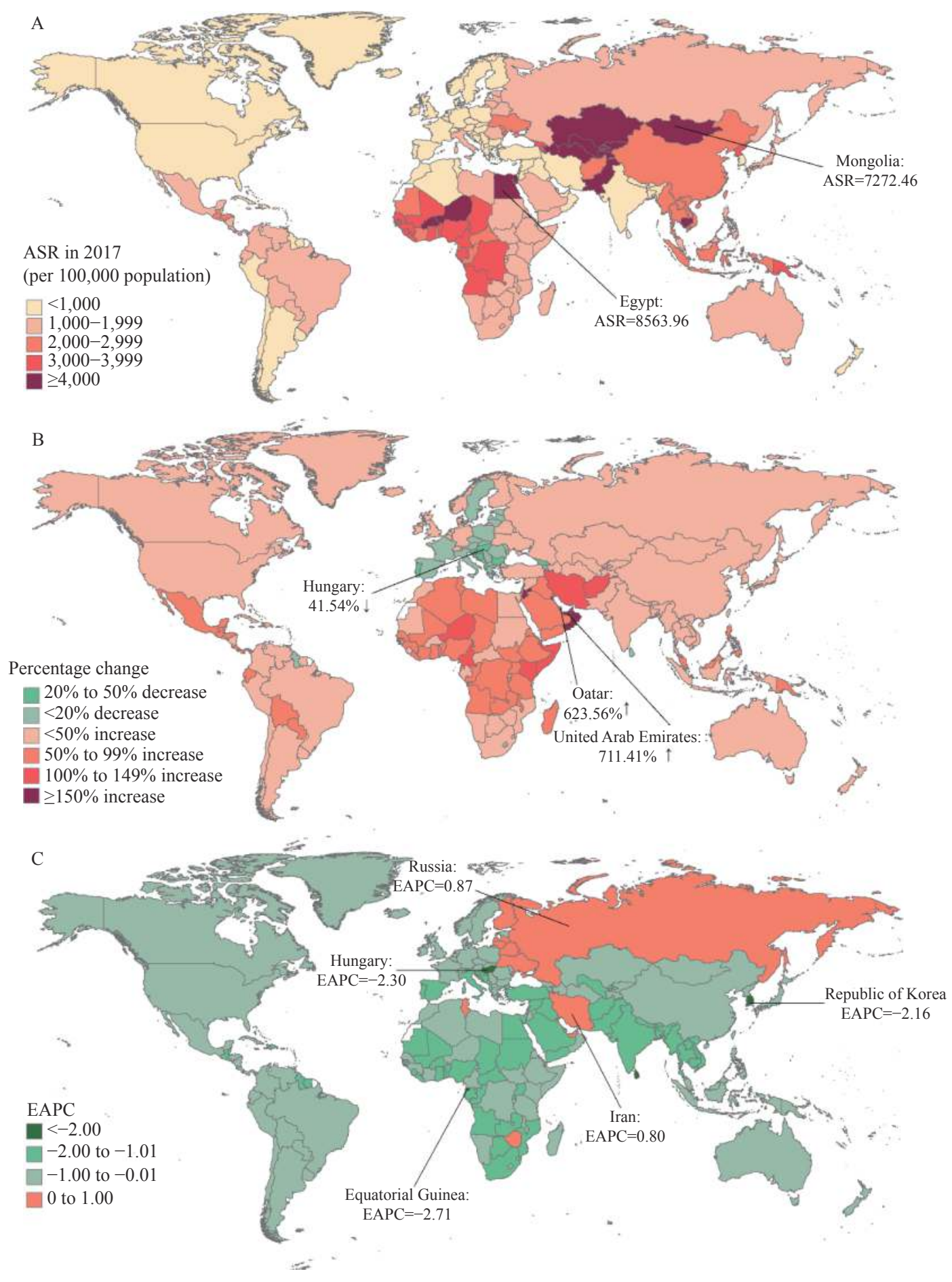

SUPPLEMENTARY FIGURE S2. The global trends of cirrhosis and other chronic liver diseases due to hepatitis C in 195 countries and territories; (A) the age-standardized prevalence rate (ASR) of cirrhosis and other chronic liver diseases due to hepatitis $C$ in 2017; (B) the percentage change in the number of cirrhosis and other chronic liver diseases due to hepatitis $C$ between 1990 and 2017; (C) the estimated annual percentage changes (EAPCs) of age-standardized prevalence rate of cirrhosis and other chronic liver diseases due to hepatitis C from 1990 to 2017. 


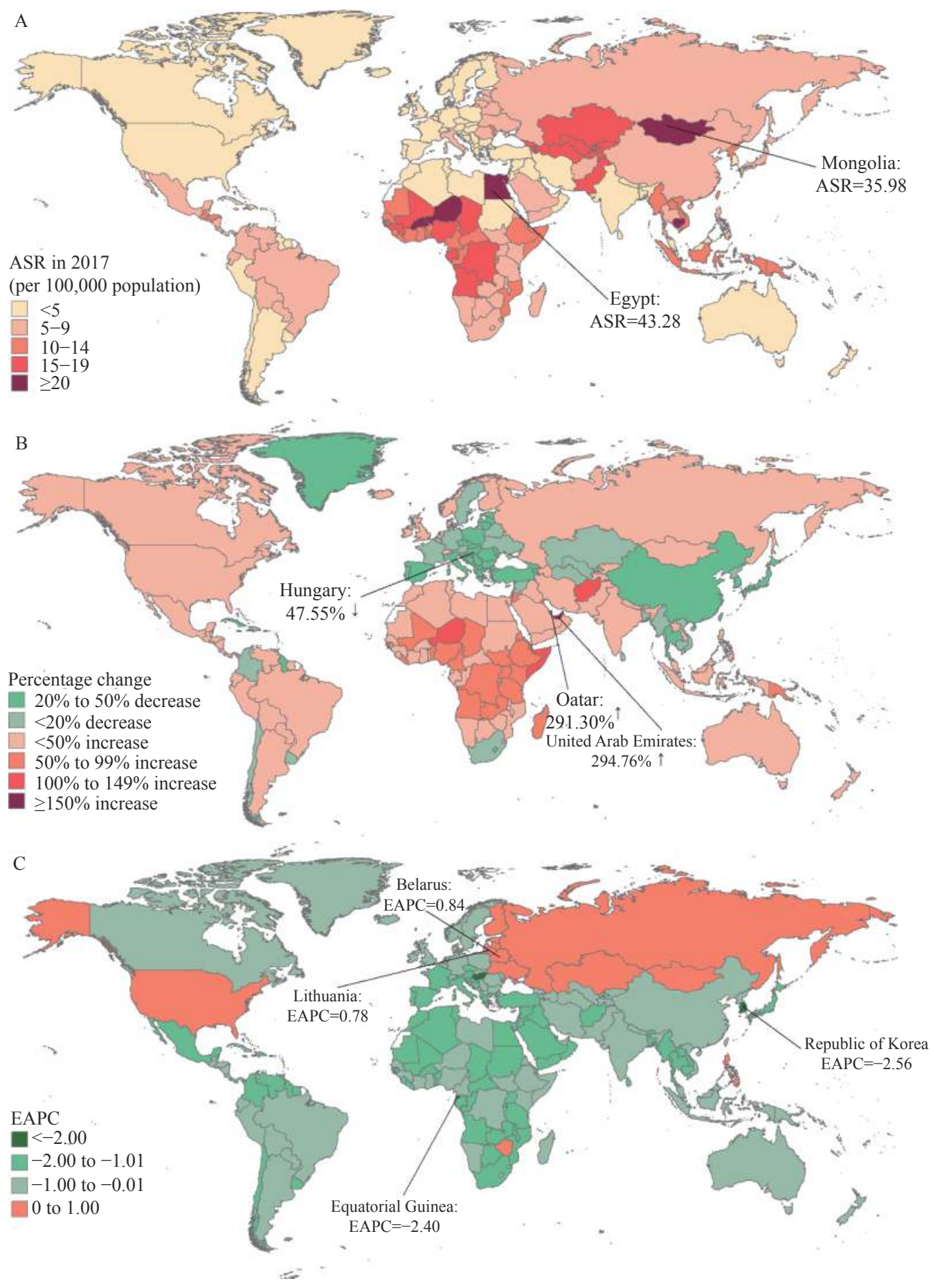

SUPPLEMENTARY FIGURE S3. The global trends of acute hepatitis $C$ in 195 countries and territories; (A) the agestandardized prevalence rate (ASR) of acute hepatitis C in 2017; (B) the percentage change in acute hepatitis C cases between 1990 and 2017; (C) the estimated annual percentage changes (EAPCs) of acute hepatitis C age-standardized prevalence rate from 1990 to 2017. 

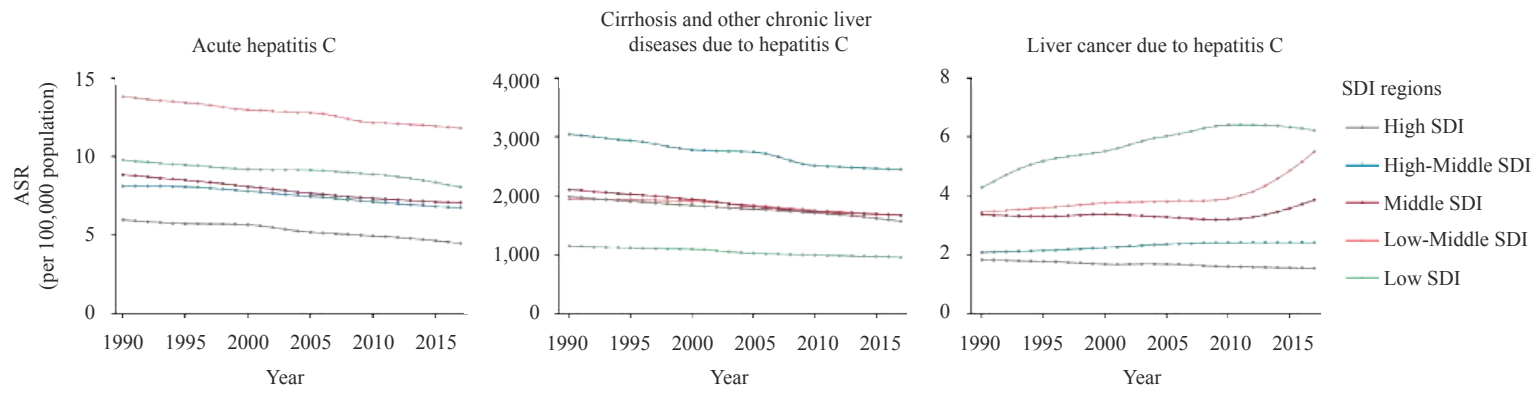

SUPPLEMENTARY FIGURE S4. The age-standardized prevalence rate (ASR) of acute hepatitis C, cirrhosis and other chronic liver diseases due to hepatitis C, and liver cancer due to hepatitis C from 1990 to 2017, by Socio-demographic Index (SDI) region. 